\title{
Short-Term Load Forecasting of Power System Based on Improved BP Neural Network
}

\author{
Sufen Li \\ School of Electrical and Electronic Engineering, Wuhan Polytechnic University, Wuhan 430023, \\ China
}

Received: May 17, 2020. Revised: November 19, 2020. Accepted: November 20, 2020.Published: November 23, 2020.

\begin{abstract}
Power system load is a stochastic and nonstationary process. Due to the influence of various factors, some bad data may exist in the load observation value. These data are mixed into the normal load data to participate in the training of neural network, which seriously affects the accuracy of load forecasting. Short-term load forecasting is the basis of power system operation and analysis, improving the precision of load forecasting is an important means to ensure the scientific decisionmaking of power system optimization. In order to improve the precision of short term load forecasting in power system, a shortterm load forecasting model based on genetic algorithm is proposed to optimize BP neural network. Firstly, using genetic algorithm to optimize the initial weights and thresholds of BP neural network to improve the prediction accuracy of BP neural network; Through the comparison and analysis before and after the model optimization, the experimental results with smaller prediction error were obtained. The simulation results show that the short-term load forecasting model established by this method has faster convergence rate and higher prediction precision.
\end{abstract}

Keywords - short-term load forecasting, genetic algorithm, BP neural network, prediction accuracy.

\section{INTRODUCTION}

$\mathrm{I}^{\mathrm{r}}$ $\mathrm{N}$ the whole power grid, the electric equipment consumes electricity, its power is load, then the power of the power system is the total load. Load curve is very important to the operation of substation, power plant and power system, it is the basis of substation load control, power plant scheduling daily generation plan, power system operation mode and main transformer, generator set and other equipment overhaul plan as well as substation and power plant extension new plan[1-3]. Load forecasting is a kind of mathematical method or model, in the case of ensuring the accuracy of the future time of the load estimates, power load forecasting on the electricity market transactions. The safety operation of the power system and the planning and design of the whole power system have influence. The prediction accuracy is closely related to the economic benefit, which is the decision basis for the power Market or department to arrange the dispatching plan, power supply demand plan and purchase and sale plan[4-5].

Therefore, the accurate prediction of power load is urgent and necessary. Load forecasting can be divided into long-term, medium-term and short-term load forecasting according to the length of forecast time and the different application situations.

Long-term load forecasting coverage time ranges from the next few years to decades, mainly used for power supply development and network development planning; mediumterm load forecasting is the forecast of electric load in the next year, which is used to arrange overhaul plan and economic operation of Reservoir. Short-term load forecasting, usually referring to future daily load forecasting and weekly load forecasting, The aim is to arrange daily and weekly power generation plans for power plants; ultra-short term load forecasting refers to load forecasting within the next 1 hours, mainly for safety monitoring, preventive control and emergency management. The medium and long term load forecasting is the precondition of developing the power system development plan, and the short-term load forecasting based on daily load curve is the basis of making the current generation plan [6]. According to the information of short-term load forecasting, we can reasonably dispatch the power generation capacity, arrange the system economy to stop and maintain the minimum cost to keep the necessary rotational reserve capacity, 
so as to minimize the cost of power generation and ensure the safe, stable, reliable, high quality and economical operation of the power grid [7]. The accuracy of short term charge prediction can help high power system personnel to make accurate and feasible electric power operation method, to ensure the safe and stable operation of power grid in various special periods, and to guarantee the economic benefit of related power enterprises, and to stabilize the power market fundamentally.

Short-term load forecasting is of great significance to the optimal combination, optimal power flow and economic dispatch of the dispatching Department, as well as to the current and future electricity market. Short-term load forecasting has become one of the important contents and research topics of power system operation, and has been paid more and more attention. For a long time, a large number of experts and scholars have carried out extensive and in-depth research, and put forward many effective methods. Compared with the conventional forecasting method, the intelligent method has great advantages and potential in establishing a unified and reasonable load forecasting model[8-10].

In recent years, more and more experts and scholars at home and abroad adopt the improved algorithm and the combination model and other prediction methods [11-18]. In paper[11], an improved Firefly algorithm (MFA) is proposed to optimize the parameters of support vector regression (SVR) model, and then to predict the short-term load. In the paper [12], researchers put forward the factors such as day type, holiday, temperature and so on in the daily load curve model, and also introduce the dynamic electricity price factor to embody the user's power demand. On the basis of this, the paper forecasts the power consumption of intelligent power grid and proves the advantage of the model effectively. In the paper [13], a new method is proposed for the dynamic demand of the users in the active distribution network, which is divided into the base load and the residual load due to the stochastic fluctuation and the demand effect. In the literatures [14-15], single models such as post propagation neural networks (BP), ARIMA, Support vector machines (SVM), which show that considering the user demand effect has the application value in the actual load forecasting. The power load is forecasted separately. In the literature [16], the original power load is decomposed by wavelet transform, and a number of calculations are carried out. Then, the load is predicted by using the finite Newton Lagrangian Support vector Machine (NLSSVM) combined with the ARIMA hybrid model. In the literature [17], the original power load is decomposed into several components by wavelet transform.

The limit learning machine and the partial least squares regression are combined to predict the components. In reference [18], the power load is decomposed into some detailed sub series by using the wavelet transform, and then the Boundary Network node (ANNA) model is used to predict each subsequence. In paper[19], the high-frequency component is filtered by wavelet transform, then the residual component is predicted by the grey Model (GM) and particle swarm optimization (PSO). Chahkoutahi et. [20] proposed a hybrid method of load forecasting based on multilayer Perceptron (MLP), Adaptive Neuro-fuzzy Inference system (ANFIS) and Sarima is proposed. N. Amjady [21] choose wavelet transform method and neural network evolutionary algorithm to deal with short-term load forecasting.

In this paper, the improved GA-BP neural network is applied to the short term load forecasting of power system, which not only has the non-linear mapping ability of BP, but also has the global searching ability of GA, which combines the two, and makes up the GA- BP neural network to predict the load. The crossover rate and mutation rate of GA are improved by adaptive algorithm, on the basis of a lot of historical data, combined with several parameters, using GA to optimize the weights and thresholds of BP neural network, the scientific and effective evaluation of short term conforming forecast by BP in the narrow range is not only simple in structure but also has strong generalization ability. The reminder of this paper is organized as follows: Section 2 provides a brief description of the BP Neural Network algorithm, followed by Genetic algorithm, and GA-BP neural network training, fitness function and encoding technique. Section 3 presents the results load forecasts, including the proposed model accuracy. These results are compared with a standard BP forecast model and SVM forcast model. The major conclusions and contributions of the paper are highlighted in Section 4.

\section{MethodologiES}

\section{A. BP Neural network}

BP Neural Network is a multilayer Feedforward network trained by error inverse propagation algorithm, it is composed of input layer, output layer and hidden layer node, which is connected only to neighboring neurons, and can be regarded as a highly parallel model composed of a group of interconnected neurons, whose performance is mainly determined by topological structure, weight value and threshold value. The training learning is divided into positive and reverse. In the process of forward propagation, the input signal is processed by the hidden layer and transmitted to the output layer, if the error between the output layer and the expected output is not up to the requirement, the error is propagated back along the original path, and the actual output and the expected output error are reduced by correcting the weights and thresholds of each connection. The design of hidden layer includes the design of hidden layer number and the design of node number of each hidden layer. Generally, the BP network with single hidden layer can map all continuous functions. Increasing the number of hidden layers can improve the accuracy and reduce the error, but also prolong the training time. Generally, the accuracy is improved by reducing the number of hidden layers and increasing the number of hidden layer nodes. A single hidden layer network with nonlinear function in hidden layer and linear function in input and output can approximate any rational function. Repeat this process continuously, reducing the entire network error to the target value. It is worth noting that an ANN with only one hidden layer can solve a problem in an optimal 
way, if the last one has the proper number of neurons.

Figure 1 is a three-layer BP neural network.

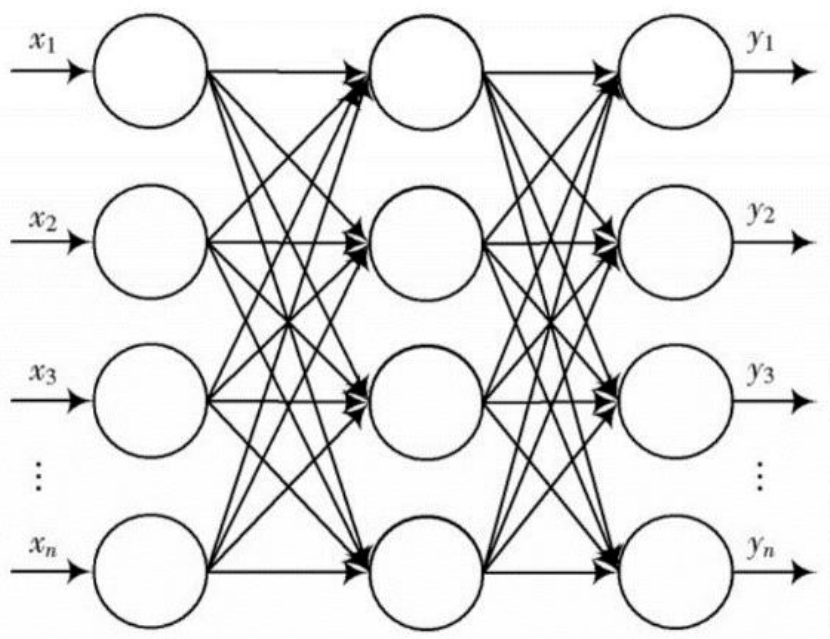

Fig. 1 Structure of three-layer BP neural network

The network can train the BP neural network through the inverse propagation of the actual output and the expected output error, and revise the weights and thresholds of the network continuously until the error of the network is minimal. The error squared between the expected output and the actual output of the network is the error objective function, i.e.

$$
\mathrm{E}_{k}=\frac{1}{2} \sum_{j=1}^{m}\left(d_{j k}-y_{j k}\right)^{2}
$$

Where, $\mathrm{m}$ is the number of output nodes of the neural network; $d_{j k}$ and $y_{j k}$ are respectively the desired output and the actual output of the neural network, the first $j k$.

\section{B. Genetic Algorithm}

Genetic algorithms use genetic manipulation and "fittest" natural selection to guide learning and determine search directions, with global search capabilities. The choice of cross probability $\mathrm{P}_{c}$ and mutation probability $\mathrm{P}_{\mathrm{m}}$ in parameters is the key to affect the behavior and performance of genetic algorithm.

But the traditional genetic algorithm chooses a solid for the crossover and mutation probabilities of all the individuals. Fixed value, the whole genetic algorithm in the process to remain unchanged. As shown in the formula (2) and formula (3).

$$
\begin{aligned}
& P_{c}= \begin{cases}P_{c 1}-\frac{\left(P_{c 1}-P_{c 2}\right)\left(f_{\max }-f^{\prime}\right)}{f_{\max }-f_{a v g}} & f^{\prime} \geq f_{a v g} \\
P_{c 1} & f^{\prime}<f_{a v g}\end{cases} \\
& P_{m}= \begin{cases}P_{m 1}-\frac{\left(P_{m 1}-P_{m 2}\right)\left(f_{\max }-f\right)}{f_{\max }-f_{a v g}} & f_{a v g} \\
P_{m} & f^{\prime}<f_{\text {avg }}\end{cases}
\end{aligned}
$$

Where, $\mathrm{P}_{\mathrm{C} 1}$ is the initial crossover probability; $\mathrm{P}_{\mathrm{C} 2}$ is the maximal value which can be increased by the crossover probability, the $f_{\max }$ is the maximal adaptive degree of the group, the $f^{\prime}$ is the larger fitness value of the cross two individual, the $f_{\text {avg }}$ is the average fitness value of each generation, the $\mathrm{P}_{\mathrm{M} 1}$ is the initial mutation probability, and $\mathrm{P}_{\mathrm{M} 2}$ is the mutation probability to increase the maximum value. In order to mutate the fitness value of the individual

\section{GA optimized BP neural network}

Although BP neural network has very strong non-linear mapping ability and simple network structure, it also has many shortcomings. For example, the algorithm convergence speed is slow and easy to fall into the local optimal solution. In this paper, the improved genetic algorithm is used to optimize the BP neural network, GA can optimize the network topology and its weights and thresholds in the process of optimizing BP neural Network, make the network model choose according to the sample knowledge, and change with the complexity of the problem, which can realize the dynamic adaptability of BP network.

GA has the advantages of global, parallelism, rapidity and good adaptability. The response and robustness can effectively solve the problem that the BP network is easily trapped in the local minima, the convergence speed is slow or not convergent. GA optimized BP neural network flowchart as shown in Figure 2. 


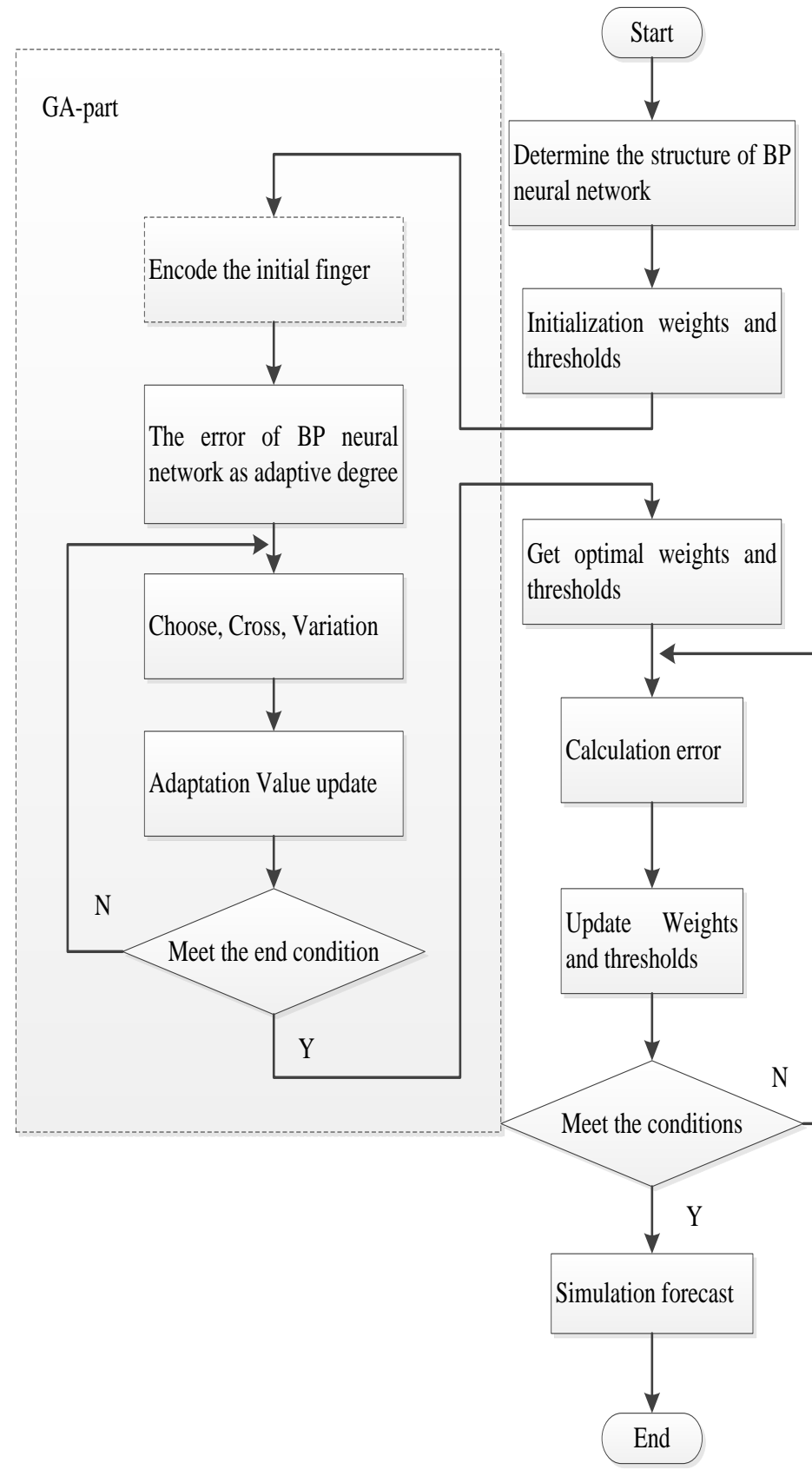

Fig. 2 Flow chart of optimization of BP neural network by GA

(1) The choice of fitness function.

The error absolute value between the predicted output and the expected output and $\mathrm{e}$ as the individual fitness values $\mathrm{F}$ are calculated as:

$$
\mathrm{F}=\mathrm{k}\left(\sum_{\mathrm{i}=1}^{n} \operatorname{abs}\left(\mathrm{y}_{\mathrm{i}}-\mathrm{o}_{\mathrm{i}}\right)\right)
$$

In the formula: $n$ is the number of network output nodes, $o_{\mathrm{i}}$ is the desired output of the node of the BP neural network, and $y_{\mathrm{i}}$ is the predictive output of the first node, and $k$ is the coefficient.

(2) Cross operation.

Because the individual uses the real number code, so the crossover operation method uses the real intersection method, the $k$ chromosome $\mathrm{a}_{\mathrm{k}}$ and the first $l$ chromosome $\mathrm{a}_{\mathrm{l}}$ in the $\mathrm{J}$-bit cross operation are as follows:

$$
\left\{\begin{array}{l}
a_{k j}=a_{k j}(1-b)+a_{1 j} b \\
a_{1 j}=a_{1 j}(1-b)+a_{k j} b
\end{array}\right.
$$

Where, $b$ is the random number of $[0,1]$.

(3) Mutation operation.

Select the J-Gene $a_{i j}$ as the first $i$ individual variation for mutation, and the mutation operation method is as follows:

$$
a_{i j}= \begin{cases}a_{i j}+\left(a_{i j}-a_{\max }\right){ }^{*} f(g) & r>0.5 \\ a_{i j}+\left(a_{m n}-a_{i j}\right){ }^{*}(g) & r \leq 0.5\end{cases}
$$

Where, $a_{\max }$ is the upper bound of the gene $a_{i j}$, the $a_{\min }$ is the lower bound of the gene $a_{i j}, r_{2}$ is a random number, $g$ is the current iteration number, the $G_{\max }$ is the maximum number of evolutions, $\mathrm{r}$ is the random number of $[0,1]$.

\section{PREDICTION EXAMPLES AND RESUlTS ANALYSIS}

The load data from March 2016 to June of a city was selected as a training sample for the June 15, 2016 to July 1. Load forecasting data based on Genetic BP neural network on June 21, 2016 are shown in Table 1. In order to compare the performance of the proposed algorithm, the traditional BP neural network method, the SVM neural network method and the GA-BP method presented in this paper are compared to predict the forecast results for 24 consecutive hours in the statistical period, and the prediction accuracy of the three is compared.

There are many methods for evaluating the performance of the prediction model. The mean absolute error (MAE) and mean standard error (MSE) are chosen as the metrics in this paper, which are as shown in (7) and (8). 


$$
\begin{array}{r}
M A E=\frac{1}{k} \sum_{i=n+1}^{n+k} \frac{\left|s_{i}-s_{i}\right|}{s_{i}} \times 100 \% \\
M S E=\sqrt{\frac{1}{k} \sum_{i=n+1}^{n+k}\left(\frac{\left|s_{i}-s_{i}\right|}{s_{i}}\right)^{2}}
\end{array}
$$

Table 1. Load forecasting data based on Genetic BP neural network on June 21, 2016 (Unit: MW)

\begin{tabular}{|c|c|c|c|}
\hline $\begin{array}{c}\text { Forecast } \\
\text { time }\end{array}$ & $\begin{array}{c}\text { Actual } \\
\text { load }\end{array}$ & $\begin{array}{c}\text { Forecast } \\
\text { load }\end{array}$ & $\begin{array}{c}\text { Relative } \\
\text { error }\end{array}$ \\
\hline $0: 00$ & 361.8000 & 361.7554 & 0.0123 \\
\hline $2: 00$ & 362.7000 & 363.9485 & 0.3442 \\
\hline $4: 00$ & 332.9000 & 343.2298 & 3.1030 \\
\hline $6: 00$ & 350.1000 & 349.7360 & 0.1040 \\
\hline $8: 00$ & 372.4000 & 372.5336 & 0.0359 \\
\hline $10: 00$ & 449.7000 & 447.5735 & 0.4729 \\
\hline $12: 00$ & 484.1000 & 479.5298 & 0.9441 \\
\hline $14: 00$ & 477.7000 & 475.2185 & 0.5195 \\
\hline $16: 00$ & 448.1000 & 458.2720 & 2.2700 \\
\hline $18: 00$ & 503.2000 & 517.2896 & 2.8000 \\
\hline $20: 00$ & 495.2000 & 504.7103 & 1.9205 \\
\hline $22: 00$ & 531.5000 & 515.2342 & 3.0604 \\
\hline $24: 00$ & 389.4000 & 390.7092 & 0.3362 \\
\hline Average relative error & & & \\
\hline Root mean square error & & & \\
\hline
\end{tabular}

In order to evaluate the applicability and superiority of the GA-BP prediction model and other predictive algorithms, a comparative analysis is given, as shown in Figure 3.

It can be seen from the graph that the absolute error of GA$\mathrm{BP}$ is smaller than the other two methods for the short term load forecasting of power system, which predicts the real value of the nearest load. Figure 4 illustrates the corresponding prediction error of different prediction algorithms, in which the error of traditional BP neural network prediction method is $3.45 \%$ to $35 \%$, and the error of standard SVM method is $1.97 \%$ to $27.5 \%$, the error distribution range of GA-BP prediction method is from $0.41 \%$ to $20 \%$. It can be clearly seen that the GA-BP has a higher predictive accuracy.

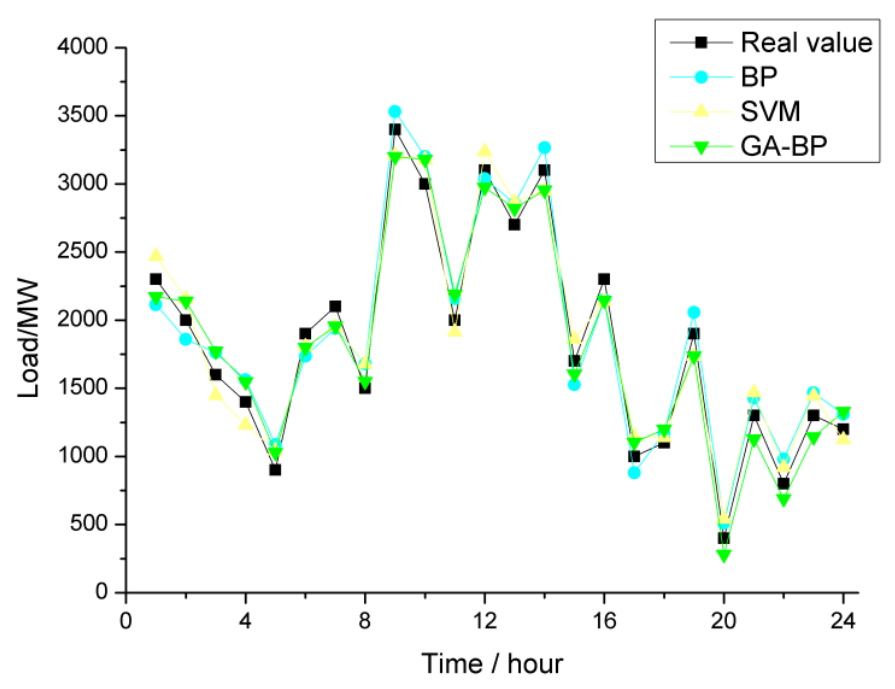

Fig.3 Measured real load and load forecasts by using different models

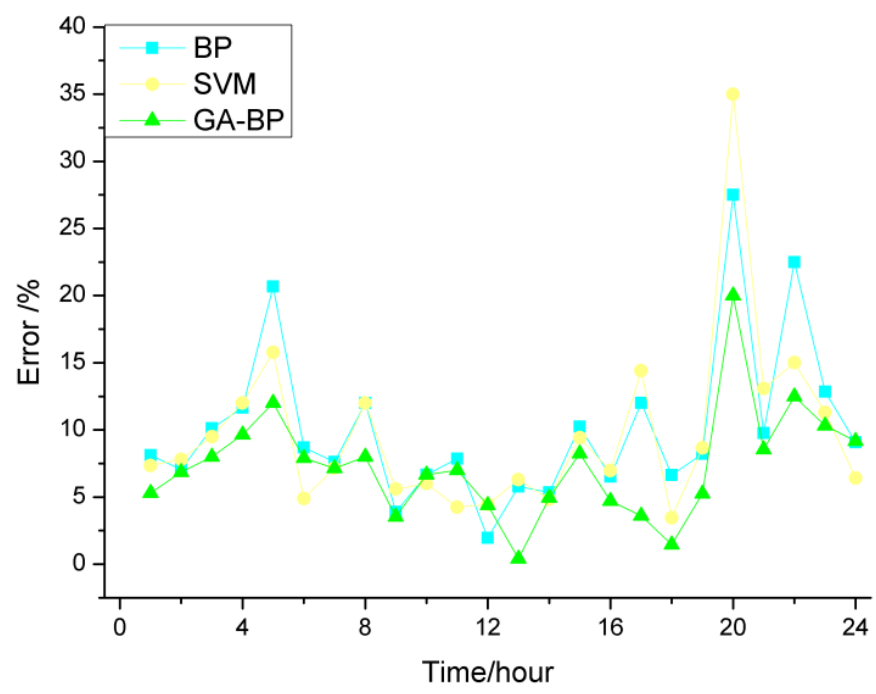

Fig.4 Prediction errors of different forecasting methods

In addition, Figure 5 and Figure 6 show the different models of Mae and MSE for one weeks. It is obvious that the proposed GA-BP method has the lowest Mae and MSE values compared to the other two models. The MSE mean ratio of GP-BP was increased by $18.2 \%$ and $16.4 \%$ respectively by standard BP neural Network and SVM neural network. Compared with standard BP neural network and SVM Neural network, the Mae mean value of GP-BP increased by $14.6 \%$ and $11.4 \%$ respectively. 


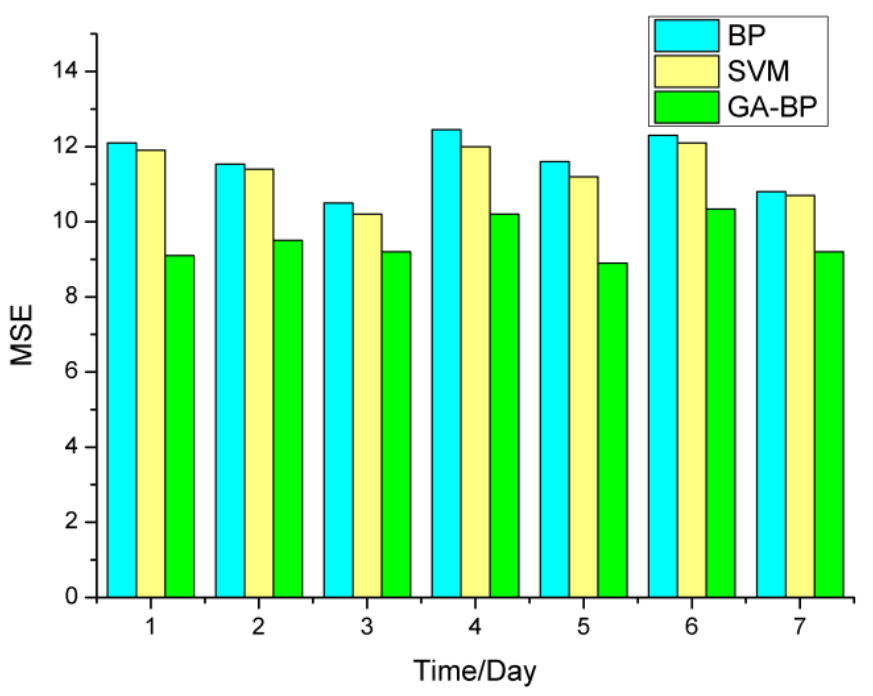

Fig.5 MSE values of different prediction methods

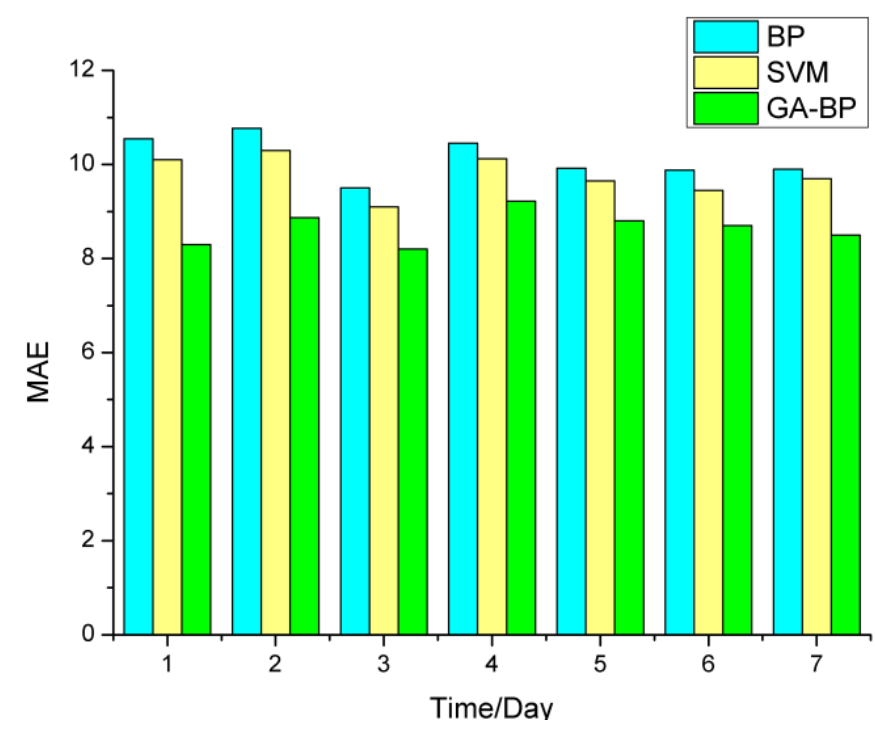

Fig.6 MSE values of different prediction methods

It is concluded that the simulation results of BP neural network model based on genetic algorithm are less error than that of BP neural network model. Compared with BP neural network model and SVM neural network model, the BP neural network model based on particle swarm optimization has higher fitting ability and prediction accuracy, and proposes a new method for short-term load forecasting.

\section{CONCLUSION}

Short-term load forecasting is the foundation of power system operation and analysis, the key research content of power system and the improvement of load forecasting precision are important means to ensure the scientific decisionmaking of power system optimization. In this paper, based on genetic algorithm optimization BP neural network for shortterm load forecasting problem modeling, will be based on genetic algorithm optimization algorithm and BP neural network combined to find more appropriate network weights and thresholds, and training, and based on genetic algorithm optimization BP model test.

The method proposed in this paper has achieved high accuracy in short-term load forecasting of power system. The training sample used in this paper is the daily load data from June 10, 2016 to June 21, 2016, and the load value on June 22, 2016 is predicted. That is to say, the summer working day model is simulated and trained. The working day model and holiday model in spring, autumn and winter have not been established yet. If it is used in practical application, the model of each season should be trained first, and the model parameters (weight, threshold, network structure parameters) should be stored in the database. In the actual use, the model parameters are called according to the model of the forecast day to forecast.

There are still some problems to be improved in this paper. For example, when the cold peak or warm peak appears for the first time, the prediction accuracy will be greatly reduced, and the prediction accuracy will be restored only after the cold peak or warm peak appears.

The author thinks that the combination forecasting is still the center of the future research work, and the validity of the load data will be the key point in the forecast work. At the same time, considering the factors such as market fluctuation, user demand, load composition, temperature and humidity of power system, introducing new model index, constructing a new model which can adapt to the law and demand of system load development, is a research idea worth further digging.

\section{REFERENCES}

[1] Cecati C, Kolbusz J, Rozycki P, et al. "A novel RBF training algorithm for short-term electric load forecasting and comparative studies", IEEE Transactions on Industrial Electronics, vol.65, no.10. pp. 6519-6529, 2015.

[2] Kale RV, Pohekar SD. "Electricity demand and supply scenarios for Maharashtra (India) for 2030: an application of long range energy alternatives planning”. Energy Policy, vol.72, no.3, pp. 1-13, 2014.

[3] Dudek G. "Pattern similarity-based methods for short-term load forecasting -Part 1: Principles". Applied Soft Computing, vol.37, no.5, pp. 285-296, 2015.

[4] Ertugrul OF. "Forecasting electricity load by a novel recurrent extreme learning $€$ machines approach". International Journal of Electrical Power \& Energy Systems, vol.78, no.5, pp.429-435, 2016.

[5] Hu R, Wen SP, Zeng ZG,et al. "A short-term power load forecasting model based on the generalized regression neural network with decreasing step fruit fly optimization algorithm". Neurocomputing, vol. 221, pp. 2431, 2017

[6] Sallan J, Villa J L, Llombart A, et al. "Optimal Design of ICPT Systems Applied to Electric Vehicle Battery Charge". IEEE Transactions on Industrial Electronics, vol.56, no.6, pp.2140-2149, 2009.

[7] Kurs A, Karalis A, Moffatt R, et al. "Wireless power transfer via strongly coupled magnetic resonances”. Science, vol.317, no.5834, pp.83-86, 2007.

[8] Song K B, Baek Y S, Hong D H, et al. "Short-term load forecasting for the holidays using fuzzy linear regression method", IEEE Transactions on Power Systems, vol.20, pp. 96-101, 2005. 
[9] Amjady N, Keynia F. "Short-term load forecasting of power systems by combination of wavelet transform and nero-evolutionary algorithm", Energy, vol.34, no.1, pp.46-67, 2009.

[10] Wang J, Zhu S, Zhang W, et al. "Combined modeling for electric load forecasting with adaptive particle swarm optimization". Energy, vol.35, no.1, pp.167-178, 2010.

[11] Abdollah K, Haidar S, Fatemeh M. "A new hybrid Modified Firefly Algorithm and Support Vector Regression model for accurate Short Term Load Forecasting", Expert Systems with Applications, vol.41, no.13, pp.6047-6056, 2014.

[12] Hosking J R M, Natarajan R, Ghosh S, et al. "Short-term forecasting of the daily load curve for residential electricity usage in the Smart Grid", Applied Stochastic Models in Business and Industry, vol.29, no.6, pp.604620, 2013.

[13] Simone P, Marco C. "Load forecasting for active distribution networks", In IEEE Pes International Conference and Exhibition on Innovative Smart Grid Technologies, 2011, pp.1-6.

[14] Xiao LY, Shao W, Yu MX, et al. "Research and application of a combined model based on multi-objective optimization for electrical load forecasting”. Energy, vol.11, no.9, pp.1057-1074, 2017.

[15] Xiao LY, Shao W, Wang C, et al. "Research and application of a hybrid model based on multi-objective optimization for electrical load forecasting", Applied Energy, vol.180, pp.213-233, 2017.

[16] Ghasemi A, Shayeghi H, Moradzadeh M,et al. "A novel hybrid algo rithm for electricity price and load forecasting in smart grids with demand side management", Applied Energy, vol.177, no.25, pp.40-59, 2016.

[17] Li S, Goel L, Wang P. "An ensemble approach for short-term load forecasting by extreme learning machine", Applied Energy, vol.170, pp.22-29, 2016.

[18] Ghofrani M, Ghayekhloo M, Arabali A, et al. "A hybrid short term load forecasting with a new input selection framework", Energy, vol.81, no.28, pp.777-786, 2017.

[19] Bahrami S, Hooshmand RA, Parastegari M. "Short term electric load forecasting by wavelet transform and grey model improved by PSO (particle swarm optimization) algorithm”. Energy, vol.72, pp. 434-442, 2017.

[20] Chahkoutahi F, Khashei M. "A seasonal direct optimal hybrid model ofcomputational intelligence and soft computing techniques for electricity load forecasting", Energy, vol.140, no.83, pp.988-1004, 2017.

[21] Amjady N, Keynia F. "Short-term load forecasting of power systems by combination of wavelet transform and neuro-evolutionary algorithm", Energy, vol.34, no.1, pp.46-57, 2009.

\section{Creative Commons Attribution License 4.0 (Attribution 4.0 International, CC BY 4.0)}

This article is published under the terms of the Creative Commons Attribution License 4.0

https://creativecommons.org/licenses/by/4.0/deed.en_US 